\title{
Postural sway during retinal image stabilisation
}

\author{
D N RUSHTON, * Th BRANDT, $\dagger$ W PAULUS, $\dagger$ S KRAFCZYK $\dagger$ \\ From the MRC Neurological Prostheses Unit, Institute of Psychiatry, London, ${ }^{*}$ UK; and Neurologische Klinik, \\ Klinikum Grosshadern, Universitat Munchen, $\uparrow$ Federal Republic of Germany
}

SUMMARY Posturographic measurements using a piezoelectric platform were made in normal subjects while wearing a combination of spectacle and contact lens providing partial stabilisation of the retinal image (RIS). The amount of postural sway seen while wearing the device at rest is intermediate between the "normal vision" and "eyes closed" conditions, and increases with increasing amounts of RIS. However, when large active head-and-eye movements are performed, postural sway is dramatically increased when using RIS, and is then worse than while performing the same task in the "eyes closed" condition. It is concluded that patients who use the partial-RIS device for the treatment of severe oscillopsia may benefit only when performing tasks in which the head is relatively still, such as reading, writing or watching TV. It is also proposed that the partial-RIS device can serve as a model in normal free-standing subjects for the postural effects of oculomotor disorders.

An optical device has recently been described ${ }^{12}$ (fig 1 , upper) which provides partial retinal image stabilisation (RIS). It was intended for use by patients with visual impairment due to continuous oscillopsia caused by nystagmus. The device also unavoidably stabilises normal eye movements such as vestibuloocular, optokinetic and saccadic movements, and therefore causes motion of the visual world during eye movements and head motion. Patients for whom the device is useful when the head is at rest find it destabilising when they move about; so do normal subjects who wear the device. Postural instability is also noted by patients with oculomotor deficits, oscillopsia caused by nystagmus, or after cataract surgery with spectacle correction. ${ }^{3}$ Such patients complain of an ataxia of visually-controlled tasks, of which postural control is an obvious example. It seems likely that the reason may be superficially similar in each case: eye movements no longer have the expected effect on retinal image position.

We decided that study of the effect of the RIS device on postural stability could serve two purposes. Firstly, it could predict what precautions should be taken by patients who wear it as a treatment for oscillopsia. Secondly, it might serve as a model for some of the destabilising effects introduced by oculomotor disease.

Address for reprint requests: D N Rushton, Medical Research Council Neurological Prostheses Unit, Institute of Psychiatry, London SE5 8AF, UK.

Received 10 May 1988 and in revised form 24 September 1988. Accepted 27 September 1988
Optical RIS offers a way of reducing to a predictable and equal extent the effect of each different class of eye movement (except that torsion movements are unaffected). It introduces an apparent loss of gain of the vestibulo-ocular reflex (VOR), with a consequent illusion of movement of the world in the oppositen direction to head rotation. There is a saccadiog hypometria (and therefore an illusion of movement in the direction of the saccade), and a marked loss of target pursuit capability (although the ocular pursuit velocity itself is greatly magnified, since smooth pursuit is a closed-loop system). Unlike visual blurring, which simply degrades visual control of posture, RIS introduces misleading information about movement of the surroundings when eye or head movements occur. At the same time, other classes of visual information relating to head position (such as parallax movements and image size changes) are enhanced, because of the linear magnification of the optical device.

\section{Methods}

Posturographic techniques ${ }^{4}$ employing a force measuring platform can show quantitatively the effect on postural sway of the introduction of different types and severity of visual limitation or degradation. We therefore used posturographic recording with the aid of such a platform, so as to obtain in normal subjects information on the nature and severity of increased postural sway caused by 0.72 RIS (the greatest RIS the device can provide in its present form); and how this varies with the visual cues available. Head and body sway were recorded simultaneously over a standard period of $25 \mathrm{~s}$, 

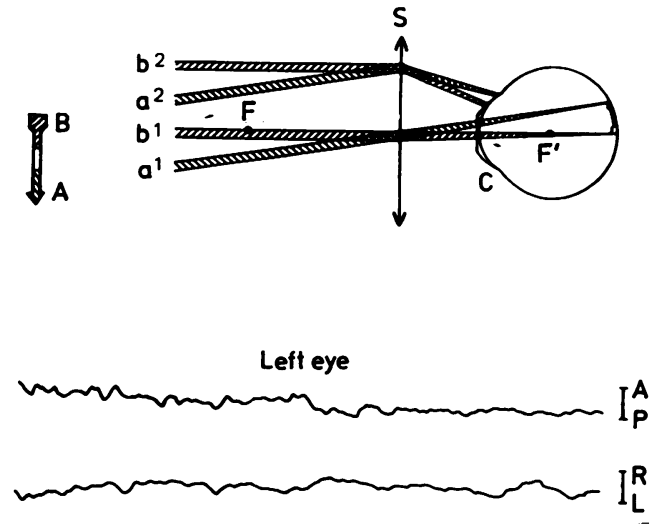

Right eye (stabilised)
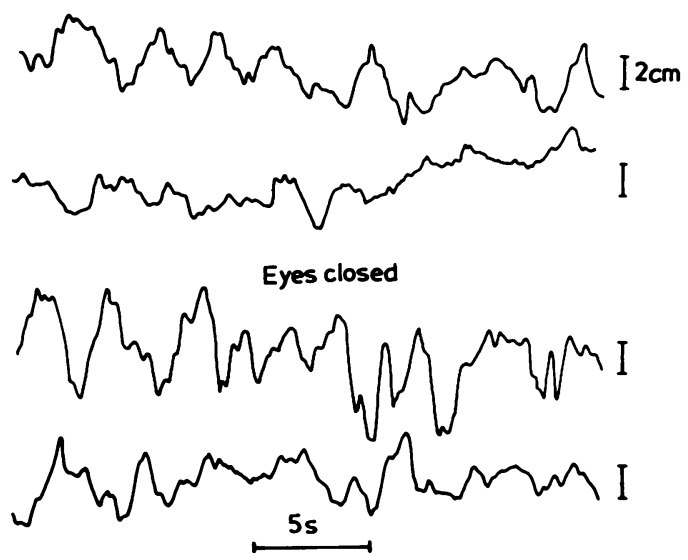

Fig 1 (a) Optical principle of the retinal image stabiliser device, which can be worn to give partially stabilised vision of the real world $(A B)$. The high-plus spectacle lens $S$, in the case of perfect stabilisation, has its principal focus $F$ at the centre of rotation of the eye, so that whichever way the eye turns, it continues to view the same point in space $(B)$. $A$ high-minus contact lens is then required, so as to restore image focus to the retina. Lower-powered lenses result in correspondingly lesser degrees of retinal image stabilisation (RIS). (b) Original recordings of fore-aft and lateral body sway (calibration mark $2 \mathrm{~cm}$ ), over a period of $25 \mathrm{~s}$ in one subject (cumulative data are given in columns $A, B$ of left side of fig 2). Body sway is markedly reduced with monocular fixation (left eye, $30^{\circ}$ restricted field, normal vision) of a stationary background $70 \mathrm{~cm}$ distant, as compared with the "eyes closed" condition. With monocular vision and 0.72 RIS ("right eye"), postural sway is intermediate between normal monocular vision and the "eyes closed" condition.

head sway by tracing an infrared light emitting diode (LED) attached to a hat worn by the subject, using a position sensor camera (Hamamatsu). Body sway was measured using a force measuring platform (Kistler).

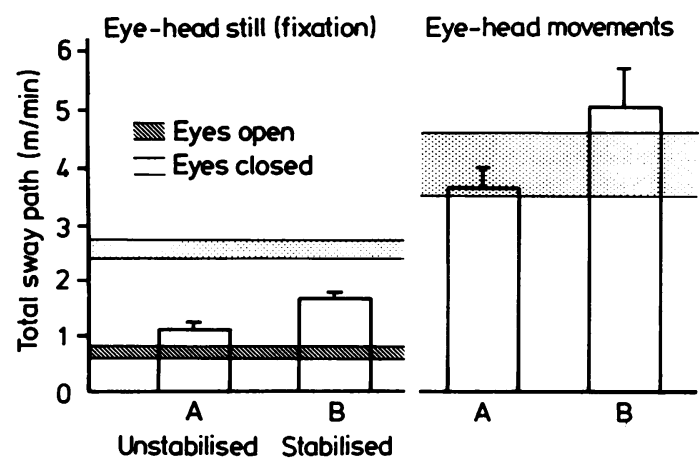

Fig 2 Total body sway path for postural balance (means and standard deviations for 10 runs: two runs in each of five subjects), with either fixation of a stationary target (left) or large combined eye-head movements (right). For fixation of a stationary target (left part of fig), the sway path is least for full-field binocular vision (striped band), and is increased (by about $50 \%$ ) for restricted-field monocular but otherwise normal vision (left, A). For restricted-field RIS vision (left, B), sway is increased by $130 \%$ over the full-field figure, while for eyes-closed (speckled band) sway is increased by $260 \%$. All of these values are significantly different from each other at $p<0.01$ level (2-tail t-test). For large eye-head movements (right), sway is greatly increased for "normal" monocular vision (A), "RIS" (B), and "eyes closed" (speckled band). However, the striking difference is the excess extra sway under the "RIS" condition, such that body sway is now significantly ( $p<0.05,2$-tail $t)$ greater even than for "eyes-closed".

The camera offered a resolution of 4,000 points in $X$ and $Y$ directions; it was attached to the ceiling above the platform. Variable lens-LED distances due to individual height differences were corrected by a personal computer. The platform contained 12 piezoelectric force transducers, one each for X, Y, Z directions at each corner. The distribution of the four $\mathrm{Z}$ forces, together with the shearing forces in $\mathrm{X}, \mathrm{Y}$ directions allow calculation of the resulting sway path (which is the length of the path traced by the centre of foot pressure), calculated in $\mathrm{m} / \mathrm{min}$. While the head sway represents net head movement (the length of the path traced by the hat-mounted LED), "body sway" as measured inevitably comprises both static sway and the superimposed dynamic force components exerted at the feet in order to keep upright. The data were digitised at $40 \mathrm{~Hz}$; the resolution of head sway was $0.5 \mathrm{~mm}$, and that of body sway $0.8 \mathrm{~mm}$.

We set out to measure the effect of RIS on postural sway with head and eyes kept still, during eye movements, and during combined eye-head movements. For some subjects, eye movements were recorded by EOG. A slab of foam rubber (height $10 \mathrm{~cm}$; specific weight $40 \mathrm{~g} / \mathrm{dm}^{3}$ ), was placed on top of the platform and covered by a second, rigid foot support. This was to reduce the somatosensory contribution to postural control and therefore to enhance the sensory weight of vision. For naive subjects, the order of the experimental conditions was randomised in case there was a learning effect in posture control under the reduced somatosensory condition. 


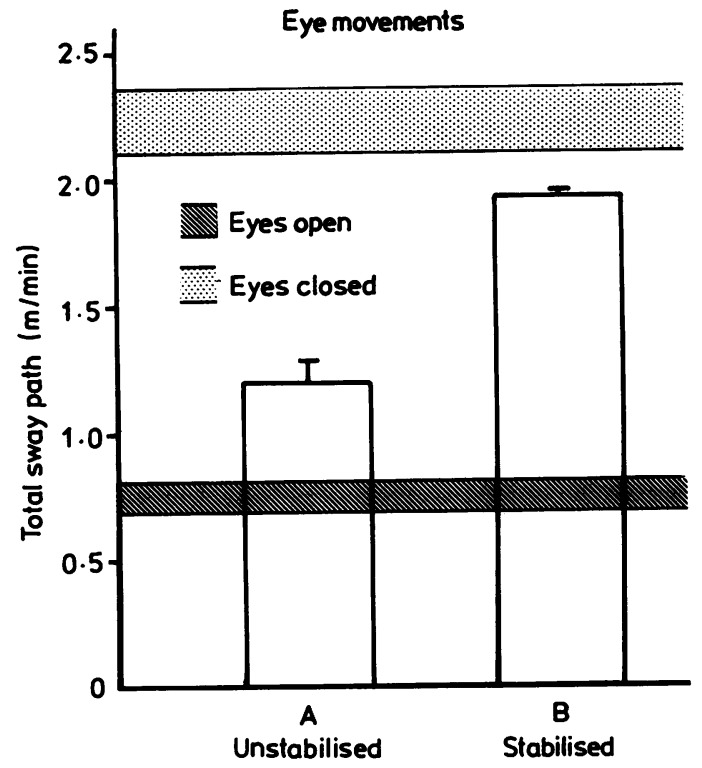

Fig 3 This illustrates the effects of horizontal saccadic eye movements, of amplitude about $\pm 15^{\circ}$, presented as in fig 2 , showing total body sway path in average of two runs in each of five subjects. The conditions are: 1 . Normal binocular vision (striped band); 2. Restricted-field "normal" monocular vision (A); 3. Restricted-field "RIS" vision (B); 4. "Eyesclosed" (speckled band). For all conditions, the amplitude of sway is similar to that seen in steady fixation (fig 2, left) and quite different from that seen in combined eye-head movements (fig 2, right).

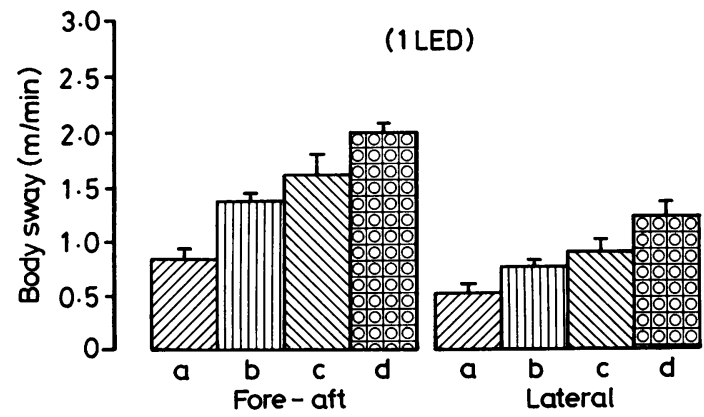

Fig 4 Fixation of a single LED in the dark. Total sway path for fore-aft and lateral body sway (Mean and $S D$ for 10 runs; two runs each in five subjects). The conditions are: (a) Left eye, restricted field, "normal" vision, full lighting, (b) Left eye, fixating one LED at $70 \mathrm{~cm}$ distance in darkness; (c) Right eye, 0.72 RIS fixating one LED at $70 \mathrm{~cm}$ in darkness; (d) Eyes closed. The results here show that "RIS" vision $(C)$ is significantly ( $p<0.05 ; 2$-tail t-test) worse than "normal" vision $(B)$, whether one considers foreaft sway (left), where the visual cues are not expected to be misleading during RIS vision, or lateral sway (right), where the visual cues available are misleading.

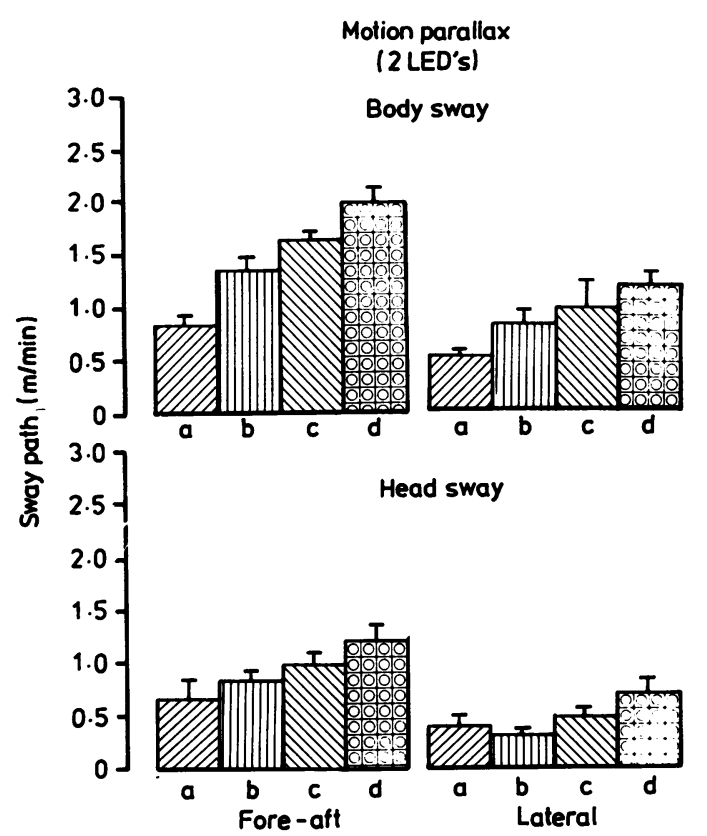

Fig 5 Fixation of two LEDs in the dark, at 35 and $70 \mathrm{~cm}$ distance in line of sight. The two-LED experiment was intended to restore the cues to lateral sway in the RIS condition, by providing parallax information which is unaffected by RIS. The conditions are otherwise as for fig 4, except that body sway (upper) and head sway (lower) are both given. Mean and SD for two runs in each of five subjects. It was found that lateral head and body sway is still greater $(p<0.05)$ for "RIS" than for "normal" monocular vision, although the parallax cue available is not misleading.

A pilot series of experiments was run using eight subjects (five naive, three non-naive), not all of whom undertook all conditions. The results reported here are taken from the main series of five RIS-naive subjects (different from the pilot series), who each undertook all conditions, except for the weak lens (RIS $=0.35$ ) experiments. The RIS $=0.35$ results mentioned in text below are therefore taken from the pilot experimental series.

The data presented from the main experiments constitute the grand average of two 25 -sec runs in each of the subjects, ages 27, 27, 25, 25 and 36 years (that is, 10 runs for each condition). They are plotted in figs 2-5 with their standard deviations. All experiments using LEDs or with the eyes closed were done in blackout; the other conditions used normal room illumination. Informed consent was obtained from all naive subjects after explanation of the procedure. No indication was given as to what results might be expected. Results from non-naive subjects did not differ from those for naive subjects, except where noted below.

\section{Results}

Fixating

The total body sway when fixating a well lit random- 
dot background monocularly with the partial-RIS device in the right eye (example fig 1, middle pair of traces) was for every subject intermediate between the "eyes closed" condition (example fig 1, lower pair of traces) and "normal monocular" vision using the left ("normal") eye (example fig 1, upper pair of traces). The "normal" eye was in fact restricted using a ring occluder mounted on a spectacle frame, so that its field covered about the same retinal extent $\left(30^{\circ}\right)$ as the RIS eye, for comparability. Means and SDs for the 10 runs in five subjects under "normal" and "RIS" conditions are given in fig 2 (left). When the head and eyes are kept still, RIS vision (B) gives sway that is intermediate between restricted field "normal" monocular vision (A) and eyes-closed (light hatched band). For comparison, the dark hatched band shows the range for normal full-field binocular vision.

\section{Combined head-and-eye movements}

However, when self-paced combined eye-and-head movements (amplitude $\pm 45^{\circ}$, velocity $30^{\circ} / \mathrm{sec}$ approx) were performed, the results were strikingly different. Performing head-and-eye free roving movements increased postural sway for all conditions (fig 2, right), but the major result here is that postural sway with RIS vision (B) is significantly $(p<0.05 ; n=10 ; 2$-tail $t$ ) worse even than when similar eye-and-head movements are made with the eyes closed (hatched band).

\section{Eye movements}

In order to determine whether it was the eye or the head component of the movements which made RIS vision so destabilising, we then studied the effect on postural sway of eye movements without head movement. The subjects performed self-paced $\pm 15^{\circ}$ horizontal saccadic eye movements at $0.5 \mathrm{~Hz}$, with the head still (fig 3). Again, the hatched bands indicate the sway for normal binocular vision without eye movements (dark hatching), and eyes closed (light hatching). It is seen that eye movements result in a moderate increase in sway both for "normal monocular" and "RIS" conditions, when compared with the fixation experiment. However, sway is here less for the "RIS" than for the "eyes closed" condition, suggesting that saccades do not make RIS actively destabilising to posture, if the head is still.

\section{Weaker partial RIS}

Some patients with nystagmus require less than 0.72 RIS in order to abolish their oscillopsia, because their oscillopsia amplitude is smaller than their amplitude of nystagmus, and their motion sensitivity is reduced. ${ }^{156}$ We therefore also recorded (pilot experiment, two subjects) the effect of 0.35 RIS. At rest, the sway path (not shown in figs) was for each subject intermediate between the results obtained for "normal" and "RIS" in fig 2. For head-and-eye free roving movements even " 0.35 RIS" was still slightly worse than "eyes-closed".

\section{Reduced visual conditions and RIS}

(a) Monocular viewing of a single LED.

Monocular fixation of a single LED in an otherwise dark room is known to reduce lateral sway in particular, ${ }^{4}$ when compared with total darkness. In this condition (fig 4), subjects were instructed to fixate the LED. Body sway during "RIS" viewing of the LED (c) was greater than for "normal" viewing $(b)$, both for lateral sway (fig 4, right) and for fore-aft sway (fig 4, left). However, this experiment does not determine whether the increased sway is due to a reduction in the sensory weight attached to vision during RIS, or due to misleading R-L cues seen during RIS vision.

(b) Monocular viewing of two LEDs

We therefore tested monocular fixation of a pair of LEDs arranged so as to provide unequivocal visual evidence of lateral sway. The LEDs were positioned at 35 and $70 \mathrm{~cm}$ in line of sight, so that they would appear to move in parallax during lateral sway. Conditions were otherwise as for the single LED experiment. Although the major visual cue for perception of lateral self-motion (parallax) is magnified for "RIS" when compared with "normal" monocular restricted view, it is seen in fig 5 (b-"normal"; c-"RIS") that lateral as well as fore-aft sway is still greater for RIS than for normal condition $(\mathrm{p}<0.05 ; \mathrm{n}=10,2$-tail $t)$.

\section{Discussion}

\section{Fixation experiments}

In this condition, RIS vision was contributing a reduction of postural sway, since sway was less than for "eyes closed". The question remained whether RIS was inferior to normal vision because of sensory conflict (particularly with the vestibular sense, since position sense had been degraded), or some other cause such as reduction in sensory weight attached to vision. We therefore attempted either to alter sensory conflict or to reduce visual sensory weight, in the subsequent experiments.

Eye movement and eye-and-head movement conditions These experiments introduced different sorts of sensory conflict. Moving only the eyes resulted in a moderate increase in sway for each visual condition; but RIS vision was still an asset, as sway was significantly less than for eyes closed. Moving eyes and head together, however, dramatically increased sway for RIS vision, so that sway was worse than when the same movements were made with eyes closed. RIS in this condition is actively destabilising posture. The 
absolute increase in sway in the eye-head movements condition is partly attributable to the necessary postural corrections needed to compensate for the head movements, and partly to the degradation of vestibular control caused by the large head movements. However, the differences between the three visual conditions are alone responsible for the differences between "monocular normal", "RIS" and "eyesclosed" sway averages in fig 2 , right. This experiment then suggests that sensory conflict is important in increasing sway, since if the effect of RIS vision on posture could be suppressed, sway would have been no worse than with eyes closed.

\section{LED experiments}

The unselective increase in both A-P and R-L sway for RIS vision under reduced visual conditions using a single LED was unexpected, since the change in angle subtended by the LED during fore-aft sway is greater for "RIS" than for the "normal" condition. The reason for this is the angular magnification (about $\times 2$ ) that is a side effect of RIS optics.' It would be expected therefore that localisation in distance should be enhanced. Localisation in the frontal plane using RIS vision is expected to be impaired owing to the illusions of object movement caused by eye movement. The result suggested that there may be a non-specific reduction in the weight attached to visual information during RIS. The 2-LED experiment tested this possibility by enhancing R-L localisation in a way that was unimpaired by RIS vision. Again, it appeared that the information in the display was not being fully used.

The LED results taken together suggest that the altered effect of eye movements in RIS may raise the threshold of perception of self-motion, or reduce the sensory weight attached to the visual signals, rather than simply introducing sensory conflict. It is worth noting that in the pilot experiments under the 2-LED condition (not shown), the one subject non-naive for RIS (DNR) did succeed (by dint of conscious attention to the parallax information) in reducing his lateral sway (but not fore-aft sway), as would be predicted if visual cues had been given their usual weight.

\section{Practical implications for RIS}

Patients who are prescribed an optical RIS device for the treatment of disabling oscillopsia should be warned that it may cause postural instability, especially during combined eye-head movements, and that it should therefore be used only for tasks performed seated where the head is relatively stationary (such as reading, writing and watching TV). Adaptation to an 0.72 stabiliser is likely to be more difficult than to an 0.35 stabiliser.
Effect of simulated oculomotor deficit on postural control

Partial RIS may also serve, as here, in normal subjects as a model for study of the perceptual and reflex effects of oculomotor deficit; it is harmless and repeatable, unlike retrobulbar injection of lignocaine or curare.

\section{Alterations in sensory weight when using RIS}

Postural control is multisensory, with integration of visual, somatosensory and vestibular signals. If the signals of one class are reduced or degraded (for example by visual blurring or standing on foam rubber), then the contribution of that pathway is reduced. There is some uncertainty ${ }^{89}$ over the effect in normal subjects when misleading visual information arrives, and vision during RIS is posturally misleading both during head and eye movements. How effectively is this misleading information suppressed? It is known that subjects with a vestibular deficit cannot suppress their destabilising responses to misleading visual information unless the angular velocity is small, ${ }^{10}$ while normal subjects adapt so as to suppress them, ${ }^{11}$ although not instantly. ${ }^{9}$ Our results suggest that when the head is still, normal subjects can suppress destabilising responses generated by eye movements during RIS, while maintaining visual information on egocentric localisation, since the head and body sway with RIS is then less than with eyes closed. However, some useful visual cues may be ignored, as is illustrated in the 2-LED condition, where naive subjects fail to utilise the relevant visual stimulus (parallax motion), which is available in RIS just as it is in normal vision.

When the vestibular control of posture is degraded by large head-and-eye movements (which also increase the misleading visual signals), then the sway with RIS is greater than in the eyes-closed condition. This suggests that the destabilising responses to misleading visual signals are not fully suppressed when vestibular signals are degraded, in normal people. Thus, the loss of visual postural response suppression may be not only a pathological phenomenon associated with vestibular failure, but may also occur in normal people when they place their vestibular system at a disadvantage.

This work was supported by the Medical Research Council and the Deutsche Forschungsgemeinschaft.

\section{References}

1 Rushton DN, Cox ND. A new optical treatment for oscillopsia. J Neurol Neurosurg Psychiatry 1987; 50:411-5.

2 Rushton DN, Rushton RH. An optical method for approximate stabilization of vision of the real world. $J$ Physiol (Lond) 1984;357:3P. 
3 Brandt Th. Visual vertigo and acrophobia. In: Dix MR, Hood JD, eds. Vertigo. New York: Wiley, 1984: 439-66.

4 Paulus W, Straube A, Brandt Th. Visual stabilization of posture: Physiological stimulus characteristics and clinical aspects. Brain 1984;107:1143-63.

5 Buchele W, Brandt Th, Degner D. Ataxia and oscillopsia in downbeat-nystagmus vertigo syndrome. Adv OtoRhino-Laryngol 1983;30:291-7.

6 Leigh RJ, Rushton DN, Thurston SE, Hertle RW, Yaniglos SS. Effects of retinal image stabilization in acquired nystagmus due to neurologic disease. Neurology 1988;38:122-7.

7 Brindley GS, Goodman GM, Kulikowski JJ, Leighton D. Stability of vision with a paralysed eye. $J$ Physiol
(Lond) 1976;258:65-66P.

8 Brandt Th, Paulus W, Straube A. Vision and Posture. In: Bles IW, Brandt Th, eds. Disorders in Posture and Gait. New York: Elsevier, 1986:157-76.

9 Vidal PP, Berthoz A, Millanvoye M. Difference between eye closure and visual stabilization in the control of posture in man. Aviat Space Environ Med 1982;53: 166-70.

10 Nashner LM, Black FO, Wall C. Adaptation to altered support and visual conditions during stance: patients with vestibular deficits. $J$ Neurosci 1982;2:536-44.

11 Nashner L, Berthoz A. Visual contribution to rapid motor responses during postural control. Brain Res 1978;150:403-7. 